The impact of financial development on income inequality: a quantile regression approach

Yener Altunbaş a, John Thornton ab*

a The Business School, Bangor University

b Office of Technical Assistance, US Department of the Treasury

* Corresponding author. Tel.: +1 202355 8637. Email:

John.Thorntondotatreas.us; j.thorntondbangor.ac.uk

\title{
ABSTRACT
}

In a panel of 121 countries, the impact of financial development on income inequality appears to change with a country's level of income. It promotes equality across inequality quantiles in uppermiddle income countries, and it promotes inequality across inequality quantiles in low- and high-income countries.

JEL: D31, D63, F02, O11, O15

Keywords: Income inequality; Financial development; Quantile regression 


\section{The impact of financial development on income inequality: a quantile regression approach ${ }^{1}$}

\section{Introduction}

Rising income inequality is a widespread concern and the impact of financial development on income inequality has received a lot of attention. Existing theories offer conflicting predictions about the impact of financial development on inequality. Greenwood and Jovanovic (1990) argue that the interaction of financial and economic development is associated with an inverted u-shaped relationship between income inequality and financial development. The rise in income in the process of development and the associated improvement in financial markets widens inequality, but as the economy matures and the financial structure is fully developed, the distribution of income stabilizes. In contrast, Galor and Zeira (1993) and Galor and Moav (2004) argue that as long as credit constraints are binding, financial development enhances economic growth and reduces inequality in non-poor economies, whereas in poor economies, modest financial development increases inequality. Moreover, in insufficiently rich economics, where credit

\footnotetext{
1 We are grateful to an anonymous referee whose comments improved the paper.
} 
constraints are less binding, financial development may increase inequality.

Existing empirical evidence provides non-conclusive findings (for surveys see, e.g., Claessens and Perotti, 2007, and de Haan and Sturm, 2017). Much of that work has been based on ordinary least squares, instrumental variables, and panel estimation, which estimate the parameters of interest at the mean evaluation by a conditional distribution of the dependent variable. In contrast, in this paper we examine the effect of financial development on income inequality using the quantile regression (QR) methodology developed by Koenker and Bassett (1978), which enables us to examine the effect at different intervals throughout the income inequality distribution. We also examine whether financial development impacts income inequality mainly through the banking sector or through financial markets, and whether there are differences in the effects across country income groups.

\section{Estimation methodology and data}

We employ the $Q R$ approach to examine the effect of financial development on income inequality but report panel fixed effects results for purposes of comparison. Using QR analysis, we can examine the determinants of income inequality throughout the 
conditional distribution, with particular focus on countries with the most equal and unequal income distributions. The quantile estimator is obtained by solving the following optimization problem:

$$
\min _{\beta \in R^{k}}\left[\sum_{i \in\left\{i: y_{i} \geq x_{i}^{\prime} \beta\right\}} \theta\left|y_{i}-x_{i}^{\prime} \beta\right|+\sum_{i \in\left\{i: y_{i}<x_{i}^{\prime} \beta\right\}} 1-\theta\left|y_{i}-x_{i}^{\prime} \beta\right|\right]
$$

for the $\theta$ th quantile $(0<\theta<1)$, where $y_{i}$ is the dependent variable and $x_{i}$ is a $k$ by 1 vector of explanatory variables.

Our dependent variable is the Gini coefficient based on households' income before taxes from Solt's (2009) Standardized World Income Inequality Database (SWIID). ${ }^{2}$ To measure financial development, we employ the index of financial development developed recently by IMF staff, which is designed to capture the depth, access and efficiency dimensions of financial institutions (banks and nonbanks) and financial markets (for explanations, see Sahay et al., 2015, and Svirydzenka, 2016), and its two key sub-indices that reflect separately the contributions to financial development from the development of financial institutions and financial markets. Our control variables are typical of the literature and

\footnotetext{
${ }^{2}$ As the SWIID standardizes incomes it facilitates comparison across countries. We follow de Haan and Sturm (2017) and use household income before taxes to proxy for income inequality before redistribution via the tax system.
} 
include: the real per capita GDP growth; the rate of inflation; the ratio of foreign trade to GDP; the ratio of government final consumption to GDP; an index of representative government to capture the impact of voter rights; and a dummy variable to control for the impact of economic crisis. ${ }^{3}$ We employ annual data for 121 countries for the period 1971-2015 with the data organized into five-year non-overlapping averages and with the independent variables lagged one (five-year) period to limit endogeneity issues.

\section{Empirical results}

Table 1 presents results for each measure of financial development for the full sample of countries. Column (1) reports the panel fixed effects estimate and columns (2) to (6) report results for the 10, 25, 50, 75 and 90 percent quantiles. Robust standard errors for the fixed effects estimates and the QR results from the 1,000 bootstrapping repetitions are reported to obtain heteroskedasticity-robust estimates. Column (6) reports the

\footnotetext{
3 Data on GDP growth, trade, and inflation are from the World Bank's World Development Indicators database. The index of representative government captures contested and inclusive popular elections for legislative and executive office and is produced by the International Institute for Democracy and Electoral Assistance. The economic crisis dummy variable ranges between 0 to 3 for each year depending upon whether a country experienced no crisis or one or more of a systemic banking crisis, a currency crisis, or a sovereign debt crisis and is based on Laeven and Valencia (2013).
} 
results of the equality test that the slope coefficients of the regression quantiles are all the same. Several observations are notable. First, the fixed effects results reported in column (1) show that all the measures of financial development have a statistically significant and negative impact on income distribution-i.e., greater financial development leads to a more unequal income distribution. Second, the impact of financial development on inequality runs through both the banking sector and through financial markets in broadly equal measures, with the coefficients on the two sub-indices positive and statistically significant. Third, the signs of the regression quantile coefficients for financial development in columns (2) to (6) are coherent with the fixed effects results and indicate that financial development is associated with an increase in income inequality at all levels of income inequality. Fourth, while financial development increases inequality at all quantile levels, its impact moderates as inequality increases-i.e., financial development increases income inequality in countries with more equal income distributions but its impact diminishes as incomes become more unequal. This is consistent with financial development providing a disproportionately larger share of access to finance to high-income groups (Claessens and Perotti, 2007). Finally, the equality test results for financial development reject the null hypothesis that all regression quantile coefficients are equal. Of 
the other explanatory variables, more representative governments appear to reduce income inequality, especially at lower levels of income inequality. However, trade openness and government consumption expenditure appear to promote income inequality with their effects increasing as incomes become more unequal. Total government consumption spending may be a poor proxy for social spending aimed at reducing income inequalities, but it may also indicate that most spending on transfers is captured by the middle class (Milanovic, 2000, Davoodi et al., 2003), which is also consistent with the quantile results. The coefficients on GDP growth and inflation are never statistically significant.

In Tables 2-4, we present results for each measure of financial development making use of the World Bank's country income classifications system. ${ }^{4}$ Table 2 reports results where total financial development is the independent variable. The results for high-income countries (panel A) and lower income countries (panel C) are in line with those for the full sample: more financial development increases income inequality at all quantile levels with its impact moderating as income inequality increases. For

\footnotetext{
4 The World Bank's classification scheme for 2015 defined low-income economies are defined as those with a GNI per capita, of $\$ 1,025$ or less in 2015; lower middle-income economies are those with a GNI per capita between \$1,026 and \$4,035; upper middle-income economies are those with a GNI per capita between $\$ 4,036$ and $\$ 12,475$; high-income economies are those with a GNI per capita of $\$ 12,476$ or more.
} 
upper-middle income countries (panel B), however, financial development has a different impact: it reduces income inequalities across quantiles, and especially at lower levels of income inequality. These results are consistent with the Galor and Zeira (1993) and Galor and Moav (2004) view of finance: in poor economies it increases the size of the small segment of society that invests in human capital and increases inequality; in middle-income economies, it reduces under-investment in human capital, which reduces income inequality; and in rich countries, though credit constraints are less binding, the marginal propensity to save increases with income so that finance permits the rich to borrow more and to increase inequality.

These conclusions broadly hold for estimates in which the development of financial institutions and of financial markets are the dependent variables, which are reported, respectively in Tables 3 and 4. In particular from these results, financial development on both measures promotes greater income inequality across quantiles for high- and lower-income countries and promotes greater equality in upper-middle income countries; and in lowerincome countries the adverse impact of financial development of income distribution appears to run mainly through the development of financial markets rather than through institutions, the 
coefficients on the latter measure not being statistically significant in the $Q R$ results (panel C, Table 3).

\section{Conclusion}

In a panel of 121 countries, the impact of financial development on income inequality appears to change with a country's level of income. It promotes equality in upper-middle income countries, and it promotes inequality in low- and high-income countries. 


\section{References}

Claessens, S., Perrotti, E., 2007. Finance and inequality: channels and evidence. Journal of Comparative Economics $35,748-773$

Davoodi, H., Tiongson, E., Asawanuchit, S., 2003. How useful are benefit incidence analyses of public expenditure and health spending? IMF Working Paper $03 / 227$.

De Haan, J., Sturm, J-E., 2017. Finance and income inequality: a review and new evidence. European Journal of Political Economy 50, 171-195.

Galor, O., Moav, O., 2004. From physical to human capital accumulation: inequality and the process of development. Review of Economic Studies 71, 1001-1026.

Galor, O., Zeira, J., 1993. Income distribution and macroeconomics. Review of Economic Studies 60, 35-52. Greenwood, J., Jovanovic, B., 1990.Financial development, growth, and the distribution of income. Journal of Political Economy 98, 1076-1107.

Koenker, R., Bassett Jr., G., 1978. Regression quantiles. Econometrica $46,33-50$.

Laeven, L., Valencia, F., 2013. Systemic banking crises database. IMF Review 61, 225-270. 
Milanovic, B., 1994. Determinants of cross-country income inequality: an "augmented" Kuznets' hypothesis. World Bank Policy Research Working Paper No. 1246.

Sahay, R., Čihák, M., N’Diaye, P., Barajas, A., Bi, R., Ayala, D., Gao, Y., Kyobe, A., Nguyen, L., Saborowski, C., Svirydzenka, K., Reza Yousefi, S.R., 2015. Rethinking Financial Deepening: Stability and Growth in Emerging Markets. IMF Staff Discussion Note 15/08. Solt, F, 2009. Standardizing the world income inequality database. Social Science Quarterly 90, 231-242. Svirydzenka, K., 2016. Introducing a New Broad-based Index of Financial Development IMF Working Paper No. 16/5. 
Table 1

Financial development and income inequality: dependent variable Gini coefficient

\begin{tabular}{|c|c|c|c|c|c|c|c|}
\hline & $\begin{array}{c}\text { (1) } \\
\text { Fixed } \\
\text { effects }\end{array}$ & $\begin{array}{l}(2) \\
Q 10\end{array}$ & $\begin{array}{c}(3) \\
225\end{array}$ & $\begin{array}{c}(4) \\
250\end{array}$ & $\begin{array}{l}(5) \\
Q 75\end{array}$ & $\begin{array}{l}(6) \\
290\end{array}$ & $\begin{array}{c}(7) \\
\text { Equality } \\
\text { test }\end{array}$ \\
\hline $\begin{array}{l}\text { Total financial } \\
\text { development }\end{array}$ & $\begin{array}{l}0.0909 * t * \\
(0.0103)\end{array}$ & $\begin{array}{l}0.1031^{* * *} \\
(0.0223)\end{array}$ & $\begin{array}{l}0.1013 * * * \\
(0.0146)\end{array}$ & $\begin{array}{l}0.0939 * * * \\
(0.0094)\end{array}$ & $\begin{array}{l}0.0851 * * \star \\
(0.0121)\end{array}$ & $\begin{array}{l}0.0805 * * * \\
(0.0154)\end{array}$ & $\begin{array}{l}3.27 \\
(0.011)\end{array}$ \\
\hline $\begin{array}{l}\text { Growth of GDP per } \\
\text { capita }\end{array}$ & $\begin{array}{l}-0.0266 \\
(0.0300)\end{array}$ & $\begin{array}{l}-0.0404 \\
(0.0778)\end{array}$ & $\begin{array}{l}-0.0556 \\
(0.0495)\end{array}$ & $\begin{array}{l}-0.0392 \\
(0.0328)\end{array}$ & $\begin{array}{l}-0.0250 \\
(0.0427)\end{array}$ & $\begin{array}{l}0.0178 \\
(0.0541)\end{array}$ & $\begin{array}{c}12.23 \\
(0.297)\end{array}$ \\
\hline Inflation & $\begin{array}{l}-0.0049 \\
(0.0064)\end{array}$ & $\begin{array}{l}-0.0006 \\
(0.0146)\end{array}$ & $\begin{array}{l}-0.0005 \\
(0.0097)\end{array}$ & $\begin{array}{l}-0.0038 \\
(0.0064)\end{array}$ & $\begin{array}{l}-0.0060 \\
(0.0083)\end{array}$ & $\begin{array}{l}-0.0074 \\
(0.0105)\end{array}$ & $\begin{array}{c}1.08 \\
(0.367)\end{array}$ \\
\hline Trade openness & $\begin{array}{l}0.0136^{* *} \\
(0.009)\end{array}$ & $\begin{array}{r}0.0028 \\
(0.157)\end{array}$ & $\begin{array}{c}0.0095 \\
(0.0103)\end{array}$ & $\begin{array}{c}0.0161 * \\
(0.0071)\end{array}$ & $\begin{array}{l}0.0217 * * \\
(0.0089)\end{array}$ & $\begin{array}{l}0.0247 * \star \\
(0.0112)\end{array}$ & $\begin{array}{c}2.22 \\
(0.064)\end{array}$ \\
\hline $\begin{array}{l}\text { Government } \\
\text { consumption }\end{array}$ & $\begin{array}{l}0.0672 * \star \\
(0.035)\end{array}$ & $\begin{array}{c}0.0717 \\
(0.0719)\end{array}$ & $\begin{array}{l}0.0648 \\
(0.0478)\end{array}$ & $\begin{array}{l}0.0747 * \star \\
(0.0317)\end{array}$ & $\begin{array}{l}0.0844 * \star \\
(0.0412)\end{array}$ & $\begin{array}{l}0.0903^{\star} \\
(0.0522)\end{array}$ & $\begin{array}{c}3.89 \\
(0.004)\end{array}$ \\
\hline $\begin{array}{l}\text { Representative } \\
\text { government }\end{array}$ & $\begin{array}{l}-0.0119 * * \\
(0.00060)\end{array}$ & $\begin{array}{l}-0.0252^{\star} \\
(0.0144)\end{array}$ & $\begin{array}{l}-0.0164 * \\
(0.0095)\end{array}$ & $\begin{array}{l}-0.0108^{\star} \\
(0.0064)\end{array}$ & $\begin{array}{l}-0.0053 \\
(0.0082)\end{array}$ & $\begin{array}{l}-0.0029 \\
(0.0104)\end{array}$ & $\begin{array}{c}1.65 \\
(0.159)\end{array}$ \\
\hline Crisis & $\begin{array}{l}0.8158 * \star * \\
(0.2455)\end{array}$ & $\begin{array}{l}1.2283 * \star \\
(0.5335)\end{array}$ & $\begin{array}{l}0.2914^{\star} \\
(0.1713)\end{array}$ & $\begin{array}{l}0.2323 * * \\
(0.1145)\end{array}$ & $\begin{array}{c}0.1911 \\
(0.1473)\end{array}$ & $\begin{array}{c}0.1655 \\
(0.1866)\end{array}$ & $\begin{array}{c}0.47 \\
(0.758)\end{array}$ \\
\hline $\begin{array}{l}\text { Observations } \\
\mathrm{R}^{2}\end{array}$ & $\begin{array}{l}794 \\
0.086\end{array}$ & $\begin{array}{l}794 \\
0.040\end{array}$ & $\begin{array}{l}794 \\
0.021\end{array}$ & $\begin{array}{l}794 \\
0.026\end{array}$ & $\begin{array}{l}794 \\
0.025\end{array}$ & $\begin{array}{l}794 \\
0.095\end{array}$ & \\
\hline $\begin{array}{l}\text { Financial } \\
\text { institutions } \\
\text { development }\end{array}$ & $\begin{array}{l}0.0704 * * * \\
(0.0134)\end{array}$ & $\begin{array}{l}0.1030 * \star * \\
(0.0317)\end{array}$ & $\begin{array}{l}0.0857 * \star \star \\
(0.0204)\end{array}$ & $\begin{array}{l}0.0697 * \star \star \\
(0.0125)\end{array}$ & $\begin{array}{l}0.0574 * * * \\
(0.0149)\end{array}$ & $\begin{array}{l}0.0513 * * * \\
(0.0186)\end{array}$ & $\begin{array}{c}3.16 \\
(0.137)\end{array}$ \\
\hline $\begin{array}{l}\text { Growth of GDP per } \\
\text { capita }\end{array}$ & $\begin{array}{l}-0.0213 \\
(0.0321)\end{array}$ & $\begin{array}{l}-0.0260 \\
(0.0891)\end{array}$ & $\begin{array}{l}-0.0543 \\
(0.0516)\end{array}$ & $\begin{array}{l}-0.0430 \\
(0.0330)\end{array}$ & $\begin{array}{l}-0.0334 \\
(0.0395)\end{array}$ & $\begin{array}{l}-0.0285 \\
(0.0493)\end{array}$ & $\begin{array}{l}1.22 \\
(0.383)\end{array}$ \\
\hline Inflation & $\begin{array}{l}-0.0046 \\
(0.0071)\end{array}$ & $\begin{array}{l}-0.0027 \\
(0.0187)\end{array}$ & $\begin{array}{c}0.0022 \\
(0.0108)\end{array}$ & $\begin{array}{l}-0.0022 \\
(0.0068)\end{array}$ & $\begin{array}{l}-0.0056 \\
(0.0082)\end{array}$ & $\begin{array}{l}-0.0073 \\
(0.0102)\end{array}$ & $\begin{array}{l}3.13 \\
(0.075)\end{array}$ \\
\hline Trade openness & $\begin{array}{l}0.0254 * * * \\
(0.0066)\end{array}$ & $\begin{array}{l}0.0078 \\
(0.0179)\end{array}$ & $\begin{array}{l}0.0200 * \\
(0.0108)\end{array}$ & $\begin{array}{l}0.0279 * \star \star \\
(0.0068)\end{array}$ & $\begin{array}{l}0.0328 * * * \\
(0.0082)\end{array}$ & $\begin{array}{l}0.0353 * \star \star \\
(0.0102)\end{array}$ & $\begin{array}{l}2.59 \\
(0.036)\end{array}$ \\
\hline $\begin{array}{l}\text { Government } \\
\text { consumption }\end{array}$ & $\begin{array}{l}0.0889 * * * \\
(0.0342)\end{array}$ & $\begin{array}{l}0.0980 \\
(0.0915)\end{array}$ & $\begin{array}{l}0.0844^{*} \\
(0.0512)\end{array}$ & $\begin{array}{l}0.0843^{* *} \\
(0.0329)\end{array}$ & $\begin{array}{l}0.0907^{* *} \\
(0.0394)\end{array}$ & $\begin{array}{l}0.0939 \star \\
(0.0491)\end{array}$ & $\begin{array}{l}1.69 \\
(0.151)\end{array}$ \\
\hline $\begin{array}{l}\text { Representative } \\
\text { government }\end{array}$ & $\begin{array}{l}-0.0074 \\
(0.0063)\end{array}$ & $\begin{array}{l}-0.0154 \\
(0.0175)\end{array}$ & $\begin{array}{l}-0.0122 \\
(0.0102)\end{array}$ & $\begin{array}{l}-0.0041 \\
(0.0065)\end{array}$ & $\begin{array}{c}0.0005 \\
(0.0078)\end{array}$ & $\begin{array}{l}0.0028 \\
(0.0097)\end{array}$ & $\begin{array}{l}1.70 \\
(0.148)\end{array}$ \\
\hline Crisis & $\begin{array}{l}0.3681 * * * \\
(0.1330)\end{array}$ & $\begin{array}{l}0.7061 * * * \\
(0.2969)\end{array}$ & $\begin{array}{r}0.3138 * \\
(0.1810)\end{array}$ & $\begin{array}{l}0.2331 * \star \\
(0.1552)\end{array}$ & $\begin{array}{c}0.1782 \\
(0.1382)\end{array}$ & $\begin{array}{r}0.01497 \\
(0.1715)\end{array}$ & $\begin{array}{l}0.40 \\
(0.812)\end{array}$ \\
\hline $\begin{array}{l}\text { Observations } \\
\mathrm{R}^{2}\end{array}$ & $\begin{array}{l}787 \\
0.115\end{array}$ & $\begin{array}{l}787 \\
0.029\end{array}$ & $\begin{array}{l}787 \\
0.022\end{array}$ & $\begin{array}{l}787 \\
0.027\end{array}$ & $\begin{array}{l}787 \\
0.029\end{array}$ & $\begin{array}{l}787 \\
0.089\end{array}$ & \\
\hline $\begin{array}{l}\text { Financial markets } \\
\text { development }\end{array}$ & $\begin{array}{l}0.0652 * \star * \\
(0.0075)\end{array}$ & $\begin{array}{l}0.0761 * * * \\
(0.0141)\end{array}$ & $\begin{array}{l}0.0710 * * * \\
(0.0102)\end{array}$ & $\begin{array}{l}0.0651 * * * \\
(0.0065)\end{array}$ & $\begin{array}{l}0.0605 * * * \\
(0.0083)\end{array}$ & $\begin{array}{l}0.0601 * * * \\
(0.0114)\end{array}$ & $\begin{array}{l}2.44 \\
(0.044)\end{array}$ \\
\hline $\begin{array}{l}\text { Growth of GDP per } \\
\text { capita }\end{array}$ & $\begin{array}{l}-0.0226 \\
(0.0305)\end{array}$ & $\begin{array}{l}-0.0388 \\
(0.0716)\end{array}$ & $\begin{array}{l}-0.0376 \\
(0.0512)\end{array}$ & $\begin{array}{l}-0.0239 \\
(0.0340)\end{array}$ & $\begin{array}{l}-0.0183 \\
(0.0433)\end{array}$ & $\begin{array}{l}-0.0273 \\
(0.0550)\end{array}$ & $\begin{array}{l}1.33 \\
(0.257)\end{array}$ \\
\hline Inflation & $\begin{array}{l}-0.0086 \\
(0.0065)\end{array}$ & $\begin{array}{l}-0.0069 \\
(0.0134)\end{array}$ & $\begin{array}{l}-0.0088 \\
(0.0098)\end{array}$ & $\begin{array}{l}-0.0066 \\
(0.0063)\end{array}$ & $\begin{array}{l}-0.0072 \\
(0.0081)\end{array}$ & $\begin{array}{l}-0.0060 \\
(0.0110)\end{array}$ & $\begin{array}{l}2.27 \\
(0.060)\end{array}$ \\
\hline Trade openness & $\begin{array}{l}0.0147 * \star \\
(0.009)\end{array}$ & $\begin{array}{c}0.0038 \\
(0.0140)\end{array}$ & $\begin{array}{c}0.0091 \\
(0.0099)\end{array}$ & $\begin{array}{l}0.0164 * * \\
(0.0065)\end{array}$ & $\begin{array}{l}0.0217 * * * \\
(0.0083)\end{array}$ & $\begin{array}{l}0.0248 * * * \\
(0.0110)\end{array}$ & $\begin{array}{c}2.46 \\
(0.044)\end{array}$ \\
\hline $\begin{array}{l}\text { Government } \\
\text { consumption }\end{array}$ & $\begin{array}{l}0.0800 * \star \\
(0.0343)\end{array}$ & $\begin{array}{c}0.0869 \\
(0.0702)\end{array}$ & $\begin{array}{l}0.0917 * \\
(0.505)\end{array}$ & $\begin{array}{l}0.0878 * \star \\
(0.0346)\end{array}$ & $\begin{array}{l}0.0892 * * \\
(0.0441)\end{array}$ & $\begin{array}{l}0.1012^{*} \\
(0.0587)\end{array}$ & $\begin{array}{l}2.01 \\
(0.092)\end{array}$ \\
\hline $\begin{array}{l}\text { Representative } \\
\text { government }\end{array}$ & $\begin{array}{l}-0.0155 * \star \\
(0.0062)\end{array}$ & $\begin{array}{l}-0.0295 * \star \\
(0.134)\end{array}$ & $\begin{array}{l}- \\
0.0252 * * * \\
(0.0096)\end{array}$ & $\begin{array}{l}-0.0134 * \star \\
(0.0064)\end{array}$ & $\begin{array}{l}-0.0069 \\
(0.0081)\end{array}$ & $\begin{array}{l}-0.0040 \\
(0.0109)\end{array}$ & $\begin{array}{c}3.22 \\
(0.012)\end{array}$ \\
\hline Crisis & $\begin{array}{l}0.7949 * * * \\
(0.2557)\end{array}$ & $\begin{array}{l}1.2610 * * * \\
(0.4778)\end{array}$ & $\begin{array}{l}1.0351 * * * \\
(0.3419)\end{array}$ & $\begin{array}{l}0.7805 * * * \\
(0.2233)\end{array}$ & $\begin{array}{l}0.5577 * \star \star \\
(0.2826)\end{array}$ & $\begin{array}{l}0.1093 * \star \star \\
(0.3840)\end{array}$ & $\begin{array}{l}1.27 \\
(0.276)\end{array}$ \\
\hline $\begin{array}{l}\text { Observations } \\
\mathrm{R}^{2}\end{array}$ & $\begin{array}{l}792 \\
0.086\end{array}$ & $\begin{array}{l}792 \\
0.029\end{array}$ & $\begin{array}{l}792 \\
0.025\end{array}$ & $\begin{array}{l}792 \\
0.034\end{array}$ & $\begin{array}{l}792 \\
0.036\end{array}$ & $\begin{array}{l}792 \\
0.045\end{array}$ & \\
\hline
\end{tabular}

Column (1) reports panel regression results with fixed country and time effects. Columns (2) to (6) contain the coefficients of quantile estimates regressions for the 10, 25, 50, 75 and 90 percent quantiles of the gini coefficient calculated as 5-year non-overlapping averages of annual data during the period 1971 to 2015The equality test applied is the F-test where the null hypothesis purports that the estimated slope coefficients for each variable are not statistically different across all the quantile estimates. The $p$-value for this test is given below the equality test value. $* * *, * *$ and ${ }^{*}$ indicate statistical significance at the $1 \%$, $5 \%$ and 10\% levels, respectively. 
Table 2

Total Financial development and income inequality by country income classification: dependent variable Gini coefficient

\begin{tabular}{|c|c|c|c|c|c|c|c|}
\hline & $\begin{array}{c}\text { (1) } \\
\text { Fixed } \\
\text { effects }\end{array}$ & $\frac{(2)}{\mathrm{Q} 10}$ & $\frac{(3)}{225}$ & $\begin{array}{ll}(4) \\
250\end{array}$ & $\frac{(5)}{275}$ & $\begin{array}{ll}(6) \\
290\end{array}$ & $\begin{array}{c}(7) \\
\text { Equality } \\
\text { test }\end{array}$ \\
\hline \multicolumn{8}{|c|}{$\begin{array}{l}\text { A. High-income countries } \\
\text { Total financial }\end{array}$} \\
\hline $\begin{array}{l}\text { Total financial } \\
\text { development }\end{array}$ & $\begin{array}{l}0.1048 * * * \\
(0.0129)\end{array}$ & $\begin{array}{l}0.1132 * * \star \\
(0.0192)\end{array}$ & $\begin{array}{l}0.1081 * * \star \\
(0.0143)\end{array}$ & $\begin{array}{l}0.1048 * * * \\
(0.0114)\end{array}$ & $\begin{array}{l}0.0927 * \star \star \\
(0.0127)\end{array}$ & $\begin{array}{l}0.0885 * * * \\
(0.0163)\end{array}$ & $\begin{array}{l}2.08 \\
(0.084)\end{array}$ \\
\hline $\begin{array}{l}\text { Growth of GDP per } \\
\text { capita }\end{array}$ & $\begin{array}{l}0.0136 \\
(0.0790)\end{array}$ & $\begin{array}{l}-0.0661 \\
(0.1263)\end{array}$ & $\begin{array}{l}-0.0467 \\
(0.0936)\end{array}$ & $\begin{array}{c}0.0134 \\
(0.0577)\end{array}$ & $\begin{array}{c}0.0123 \\
(0.0831)\end{array}$ & $\begin{array}{c}0.0282 \\
(0.1074)\end{array}$ & $\begin{array}{l}3.25 \\
(0.013)\end{array}$ \\
\hline Inflation & $\begin{array}{l}-\overline{0.0354 * * *} \\
(0.0115)\end{array}$ & $\begin{array}{l}-0.0193 \\
(0.0193)\end{array}$ & $\begin{array}{l}-0.0220 \\
(0.0143)\end{array}$ & $\begin{array}{l}- \\
0.0354 * * * \\
(0.0094)\end{array}$ & $\begin{array}{l}-0.0302 * \star \\
(0.0127)\end{array}$ & $\begin{array}{l}-0.0324 * * \\
(0.0164)\end{array}$ & $\begin{array}{l}1.86 \\
(0.118)\end{array}$ \\
\hline Trade openness & $\begin{array}{l}0.0316 * * \star \\
(0.0088)\end{array}$ & $\begin{array}{r}0.0303 \\
(0.147)\end{array}$ & $\begin{array}{l}0.0308 * * * \\
(0.0109)\end{array}$ & $\begin{array}{l}0.0316 * * * \\
(0.0076)\end{array}$ & $\begin{array}{l}0.0321 * * * \\
(0.0097)\end{array}$ & $\begin{array}{l}0.0325 * * * \\
(0.0125)\end{array}$ & $\begin{array}{l}0.13 \\
(0.969)\end{array}$ \\
\hline Government spending & $\begin{array}{c}0.1597 \star \\
(0.0831)\end{array}$ & $\begin{array}{c}0.0861 \\
(0.1397)\end{array}$ & $\begin{array}{l}0.0957 \\
(0.1035)\end{array}$ & $\begin{array}{l}0.1596 * * \\
(0.0741)\end{array}$ & $\begin{array}{l}0.1248 \\
(0.0919)\end{array}$ & $\begin{array}{c}0.1326 \\
(0.1188)\end{array}$ & $\begin{array}{l}3.68 \\
(0.006)\end{array}$ \\
\hline $\begin{array}{l}\text { Representative } \\
\text { government }\end{array}$ & $\begin{array}{l}-0.0053 \\
(0.0141)\end{array}$ & $\begin{array}{l}-0.0227 \\
(0.0247)\end{array}$ & $\begin{array}{l}-0.0190 \\
(0.0183)\end{array}$ & $\begin{array}{l}-0.0053 \\
(0.0116)\end{array}$ & $\begin{array}{l}-0.0079 \\
(0.0162)\end{array}$ & $\begin{array}{l}-0.0049 \\
(0.0210)\end{array}$ & $\begin{array}{l}0.83 \\
(0.506)\end{array}$ \\
\hline Crisis & $\begin{array}{l}0.9523 * * * \\
(0.4224)\end{array}$ & $\begin{array}{l}1.0121 \\
(0.6883)\end{array}$ & $\begin{array}{l}1.0444^{\star *} \\
(0.5101)\end{array}$ & $\begin{array}{l}0.9509 * \star \\
(0.3854)\end{array}$ & $\begin{array}{l}1.1428 * \star \\
(0.4528)\end{array}$ & $\begin{array}{l}1.1692 \star \star \\
(0.5855)\end{array}$ & $\begin{array}{c}0.37 \\
(0.827)\end{array}$ \\
\hline Observations & 262 & 262 & 262 & 262 & 262 & 262 & \\
\hline $\mathrm{R}^{2}$ & 0.102 & 0.039 & 0.101 & 0.067 & 0.044 & 0.049 & \\
\hline B. Upper middle-income & countries & & & & & & \\
\hline $\begin{array}{l}\text { Total financial } \\
\text { development }\end{array}$ & $\begin{array}{l}-0.0689 * * \\
(0.0275)\end{array}$ & $\begin{array}{l}-0.1026 * \star \\
(0.0439)\end{array}$ & $\begin{array}{l}- \\
0.0942 * * * \\
(0.0292)\end{array}$ & $\begin{array}{l}- \\
0.0860 * * * \\
(0.0199)\end{array}$ & $\begin{array}{l}- \\
0.0771 * \star \star \\
(0.0258)\end{array}$ & $\begin{array}{l}-0.0728 * \star \\
(0.0330)\end{array}$ & $\begin{array}{c}1.29 \\
(0.276)\end{array}$ \\
\hline $\begin{array}{l}\text { Growth of GDP per } \\
\text { capita }\end{array}$ & $\begin{array}{c}0.0473 \\
(0.0451)\end{array}$ & $\begin{array}{l}-0.0037 \\
(0.0836)\end{array}$ & $\begin{array}{c}0.0221 \\
(0.0605)\end{array}$ & $\begin{array}{c}0.0199 \\
(0.0412)\end{array}$ & $\begin{array}{c}0.0175 \\
(0.0536)\end{array}$ & $\begin{array}{c}0.0164 \\
(0.0685)\end{array}$ & $\begin{array}{l}3.03 \\
(0.019)\end{array}$ \\
\hline Inflation & $\begin{array}{l}0.0039 \\
(0.0099)\end{array}$ & $\begin{array}{c}0.0048 \\
(0.0203)\end{array}$ & $\begin{array}{c}0.0053 \\
(0.0149)\end{array}$ & $\begin{array}{c}0.0066 \\
(0.0102)\end{array}$ & $\begin{array}{c}0.0080 \\
(0.0132)\end{array}$ & $\begin{array}{c}0.0086 \\
(0.0169)\end{array}$ & $\begin{array}{l}0.72 \\
(0.579)\end{array}$ \\
\hline Trade openness & $\begin{array}{l}0.0290 \star \star \\
(0.0144)\end{array}$ & $\begin{array}{l}-0.0257 \\
(0.0232)\end{array}$ & $\begin{array}{l}-0.0161 \\
(0.0166)\end{array}$ & $\begin{array}{l}-0.0055 \\
(0.0114)\end{array}$ & $\begin{array}{l}0.0059 \\
(0.0146)\end{array}$ & $\begin{array}{l}0.0115 \\
(0.0187)\end{array}$ & $\begin{array}{l}1.18 \\
(0.323)\end{array}$ \\
\hline Government spending & $\begin{array}{l}0.2127 \star \star \star \\
(0.0688)\end{array}$ & $\begin{array}{l}0.2013^{\star} \\
(0.1185)\end{array}$ & $\begin{array}{l}0.2383 * \star \star \\
(0.0885)\end{array}$ & $\begin{array}{l}0.2386 * * * \\
(0.0602)\end{array}$ & $\begin{array}{l}0.2389 * * \star \\
(0.0784)\end{array}$ & $\begin{array}{l}0.2390 * * \\
(0.1001)\end{array}$ & $\begin{array}{l}1.32 \\
(0.263)\end{array}$ \\
\hline $\begin{array}{l}\text { Representative } \\
\text { government }\end{array}$ & $\begin{array}{l}0.0208 * \\
(0.0112)\end{array}$ & $\begin{array}{l}0.0475^{\star} \\
(0.0252)\end{array}$ & $\begin{array}{l}0.0334 * * \\
(0.0169)\end{array}$ & $\begin{array}{l}0.0313 * * * \\
(0.0115)\end{array}$ & $\begin{array}{l}0.0289 * \\
(0.0150)\end{array}$ & $\begin{array}{l}0.0278 \\
(0.0192)\end{array}$ & $\begin{array}{l}1.55 \\
(0.190)\end{array}$ \\
\hline Crisis & $\begin{array}{l}0.3509 * \\
(0.1963)\end{array}$ & $\begin{array}{c}0.3763 \\
(0.3345)\end{array}$ & $\begin{array}{c}0.7149 \\
(0.4786)\end{array}$ & $\begin{array}{l}0.5056 \\
(0.3271)\end{array}$ & $\begin{array}{c}0.2797 \\
(0.4237)\end{array}$ & $\begin{array}{c}0.1697 \\
(0.5406)\end{array}$ & $\begin{array}{l}1.01 \\
(0.405)\end{array}$ \\
\hline Observations & 222 & 222 & 222 & 222 & 222 & 222 & \\
\hline $\mathrm{R}^{2}$ & 0.098 & 0.067 & 0.071 & 0.078 & 0.059 & 0.040 & \\
\hline C. Lower- and lower-mic & dle-income & countries & & & & & \\
\hline $\begin{array}{l}\text { Total financial } \\
\text { development }\end{array}$ & $\begin{array}{l}0.1156 * \star \star \\
(0.0371)\end{array}$ & $\begin{array}{l}0.1352 * \star \\
(0.0687)\end{array}$ & $\begin{array}{l}0.1210 * * \\
(0.0498)\end{array}$ & $\begin{array}{l}0.0988 * * * \\
(0.0313)\end{array}$ & $\begin{array}{l}0.0717 * \star \\
(0.0357)\end{array}$ & $\begin{array}{l}0.1174 * * \\
(0.0573)\end{array}$ & $\begin{array}{l}1.51 \\
(0.200)\end{array}$ \\
\hline $\begin{array}{l}\text { Growth of GDP per } \\
\text { capita }\end{array}$ & $\begin{array}{l}-0.0383 \\
(0.0482)\end{array}$ & $\begin{array}{l}-0.0925 \\
(0.1274)\end{array}$ & $\begin{array}{l}-0.0701 \\
(0.0922)\end{array}$ & $\begin{array}{l}-0.0349 \\
(0.0580)\end{array}$ & $\begin{array}{l}-0.0150 \\
(0.658)\end{array}$ & $\begin{array}{l}-0.0143 \\
(0.1101)\end{array}$ & $\begin{array}{l}1.48 \\
(0.207)\end{array}$ \\
\hline Inflation & $\begin{array}{c}0.0007 \\
(0.0118)\end{array}$ & $\begin{array}{l}0.0302 \\
(0.0325)\end{array}$ & $\begin{array}{c}0.0220 \\
(0.0235)\end{array}$ & $\begin{array}{c}0.0092 \\
(0.0148)\end{array}$ & $\begin{array}{l}-0.0037 \\
(0.0166)\end{array}$ & $\begin{array}{l}-0.0093 \\
(0.0235)\end{array}$ & $\begin{array}{l}1.32 \\
(0.263)\end{array}$ \\
\hline Trade openness & $\begin{array}{l}-0.0087 \\
(0.0115)\end{array}$ & $\begin{array}{l}-0.0030 \\
(0.0202)\end{array}$ & $\begin{array}{l}-0.0013 \\
(0.0146)\end{array}$ & $\begin{array}{c}0.0013 \\
(0.0092)\end{array}$ & $\begin{array}{c}0.0009 \\
(0.0105)\end{array}$ & $\begin{array}{l}-0.00071 \\
(0.0173)\end{array}$ & $\begin{array}{l}1.21 \\
(0.305)\end{array}$ \\
\hline Government spending & $\begin{array}{l}0.0183 \\
(0.0409)\end{array}$ & $\begin{array}{l}-0.0041 \\
(0.0880)\end{array}$ & $\begin{array}{c}0.0041 \\
(0.0637)\end{array}$ & $\begin{array}{l}0.0168 \\
(0.0400)\end{array}$ & $\begin{array}{l}0.0288 \\
(0.0445)\end{array}$ & $\begin{array}{c}0.0299 \\
(0.0734)\end{array}$ & $\begin{array}{l}2.63 \\
(0.034)\end{array}$ \\
\hline $\begin{array}{l}\text { Representative } \\
\text { government }\end{array}$ & $\begin{array}{l}-0.0181 * * \\
(0.0086)\end{array}$ & $\begin{array}{l}-0.0451 * * \\
(0.0216)\end{array}$ & $\begin{array}{l}-0.0397 \star \star \\
(0.0156)\end{array}$ & $\begin{array}{l}-0.0313 * * \\
(0.0099)\end{array}$ & $\begin{array}{l}-0.0244^{* \star} \\
(0.0132)\end{array}$ & $\begin{array}{l}-0.0061 \\
(0.0175)\end{array}$ & $\begin{array}{l}2.32 \\
(0.570)\end{array}$ \\
\hline Crisis & $\begin{array}{c}0.1956 \\
(0.2025)\end{array}$ & $\begin{array}{c}0.2888 \\
(0.3797)\end{array}$ & $\begin{array}{c}0.2748 \\
(0.2748)\end{array}$ & $\begin{array}{c}0.2505 \\
(0.1724)\end{array}$ & $\begin{array}{c}0.0257 \\
(0.1936)\end{array}$ & $\begin{array}{c}0.1890 \\
(0.3382)\end{array}$ & $\begin{array}{c}0.25 \\
(0.909)\end{array}$ \\
\hline Observations & 310 & 310 & 310 & 310 & 310 & 310 & \\
\hline $\mathrm{R}^{2}$ & 0.071 & 0.081 & 0.033 & 0.023 & 0.107 & 0.017 & \\
\hline
\end{tabular}

Countries are classified according to the World Bank's 2015 income group classification. Column (1) reports panel regression results with fixed country and time effects. Columns (2) to (6) contain the coefficients of quantile estimates regressions for the 10, 25, 50, 75 and 90 percent quantiles of the gini coefficient calculated as 5-year non-overlapping averages of annual data during the period 1971 to 2015The equality test applied is the F-test where the null hypothesis purports that the estimated slope coefficients for each variable are not statistically different 
across all the quantile estimates. The p-value for this test is given below the equality test value. $* * *, * *$ and $*$ indicate statistical significance at the $1 \%, 5 \%$ and $10 \%$ levels, respectively. 
Table 3

Financial institutions development and income inequality by country income classification: dependent variable Gini coefficient

\begin{tabular}{|c|c|c|c|c|c|c|c|}
\hline & $(1)$ & $(2)$ & $(3)$ & $(4)$ & $(5)$ & $(6)$ & $(7)$ \\
\hline & $\begin{array}{c}\text { Fixed } \\
\text { effects }\end{array}$ & Q10 & Q25 & Q50 & Q75 & 290 & $\begin{array}{c}\text { Equality } \\
\text { test }\end{array}$ \\
\hline \multicolumn{8}{|l|}{ A. High-income countries } \\
\hline Financial & & & & & & & 2.87 \\
\hline $\begin{array}{l}\text { institutions } \\
\text { development }\end{array}$ & $\begin{array}{l}0.1124 * \star * \\
(0.0168)\end{array}$ & $\begin{array}{l}0.1310 * * \star \\
(0.0268)\end{array}$ & $\begin{array}{l}0.1234 * \star \star \\
(0.0211)\end{array}$ & $\begin{array}{l}0.1116 * \star \star \\
(0.0155)\end{array}$ & $\begin{array}{l}0.0934 * \star \star \\
(0.0186)\end{array}$ & $\begin{array}{l}0.0954 * \star \star \\
(0.0216)\end{array}$ & $(0.024)$ \\
\hline $\begin{array}{l}\text { Growth of GDP per } \\
\text { capita }\end{array}$ & $\begin{array}{c}0.0576 \\
(0.0923)\end{array}$ & $\begin{array}{c}0.0454 \\
(0.1408)\end{array}$ & $\begin{array}{c}0.0488 \\
(0.1101)\end{array}$ & $\begin{array}{c}0.0845 \\
(0.0654)\end{array}$ & $\begin{array}{c}0.0426 \\
(0.0970)\end{array}$ & $\begin{array}{c}0.0489 \\
(0.1164)\end{array}$ & $\begin{array}{c}4.33 \\
(0.002)\end{array}$ \\
\hline Inflation & $\begin{array}{l}- \\
0.0261 * * * \\
(0.0121)\end{array}$ & $\begin{array}{l}-0.0184 \\
(0.0218)\end{array}$ & $\begin{array}{l}-0.0201 \\
(0.0171)\end{array}$ & $\begin{array}{l}- \\
0.0323 * * * \\
(0.0109)\end{array}$ & $\begin{array}{l}-0.0269 * \\
(0.0151)\end{array}$ & $\begin{array}{l}-0.0269 \\
(0.0191)\end{array}$ & $\begin{array}{l}1.57 \\
(0.184)\end{array}$ \\
\hline Trade openness & $\begin{array}{l}0.0478 * * * \\
(0.0091)\end{array}$ & $\begin{array}{l}0.0377 * \star \\
(0.0154)\end{array}$ & $\begin{array}{l}0.0403 * * * \\
(0.0121)\end{array}$ & $\begin{array}{l}0.0444 * * * \\
(0.0084)\end{array}$ & $\begin{array}{l}0.0502 * \star * \\
(0.0106)\end{array}$ & $\begin{array}{l}0.0517 * * * \\
(0.0125)\end{array}$ & $\begin{array}{l}0.73 \\
(0.572)\end{array}$ \\
\hline Government spending & $\begin{array}{l}0.2551 * * * \\
(0.0864)\end{array}$ & $\begin{array}{l}0.2756^{\star} \\
(0.1413)\end{array}$ & $\begin{array}{r}0.2630 * \star \\
(0.1110))\end{array}$ & $\begin{array}{l}0.2962 * \star * \\
(0.0755)\end{array}$ & $\begin{array}{l}0.2129 * \star \\
(0.0978)\end{array}$ & $\begin{array}{r}0.1963^{\star} \\
(0.1172)\end{array}$ & $\begin{array}{c}1.49 \\
(0.205)\end{array}$ \\
\hline $\begin{array}{l}\text { Representative } \\
\text { government }\end{array}$ & $\begin{array}{l}-0.0075 \\
(0.0128)\end{array}$ & $\begin{array}{l}-0.0119 \\
(0.0264)\end{array}$ & $\begin{array}{l}-0.0098 \\
(0.0108)\end{array}$ & $\begin{array}{l}0.0053 \\
(0.0132)\end{array}$ & $\begin{array}{l}-0.0012 \\
(0.0183)\end{array}$ & $\begin{array}{c}0.0001 \\
(0.0218)\end{array}$ & $\begin{array}{c}0.16 \\
(0.960)\end{array}$ \\
\hline Crisis & $\begin{array}{l}0.5615^{* \star} \\
(0.2689)\end{array}$ & $\begin{array}{c}1.4170 \\
(0.7220)\end{array}$ & $\begin{array}{l}1.3804 * \star \\
(0.5673)\end{array}$ & $\begin{array}{l}1.2476 * * \\
(0.4157)\end{array}$ & $\begin{array}{l}1.3447 * \star \\
(0.4997)\end{array}$ & $\begin{array}{l}1.2177 * * \\
(0.6004)\end{array}$ & $\begin{array}{c}0.81 \\
(0.520)\end{array}$ \\
\hline Observations & 260 & 260 & 260 & 260 & 260 & 260 & \\
\hline $\mathrm{R}^{2}$ & 0.091 & 0.141 & 0.070 & 0.109 & 0.104 & 0.104 & \\
\hline \multicolumn{8}{|c|}{ B. Upper middle-income countries } \\
\hline Financial & - & $-0.0634 *$ & - & - & $-0.0556 *$ & -0.0470 & 1.89 \\
\hline $\begin{array}{l}\text { institutions } \\
\text { development }\end{array}$ & $\begin{array}{l}0.0745 * * \star \\
(0.0260)\end{array}$ & $(0.0385)$ & $\begin{array}{l}0.0954 * * * \\
(0.0371)\end{array}$ & $\begin{array}{l}0.0581 * \star \star \\
(0.0249)\end{array}$ & $(0.0308)$ & $(0.0307)$ & $(0.114)$ \\
\hline $\begin{array}{l}\text { Growth of GDP per } \\
\text { capita }\end{array}$ & $\begin{array}{c}0.0355 \\
(0.0448)\end{array}$ & $\begin{array}{c}0.0406 \\
(0.0781)\end{array}$ & $\begin{array}{c}0.0462 \\
(0.0728)\end{array}$ & $\begin{array}{l}-0.0028 \\
(0.0467)\end{array}$ & $\begin{array}{c}0.0155 \\
(0.0609)\end{array}$ & $\begin{array}{c}0.0128 \\
(0.0657)\end{array}$ & $\begin{array}{c}0.16 \\
(0.958)\end{array}$ \\
\hline Inflation & $\begin{array}{l}-0.0003 \\
(0.0103)\end{array}$ & $\begin{array}{l}-0.0018 \\
(0.0207)\end{array}$ & $\begin{array}{c}0.0040 \\
(0.0162)\end{array}$ & $\begin{array}{l}-0.0032 \\
(0.0108)\end{array}$ & $\begin{array}{l}-0.0056 \\
(0.0143)\end{array}$ & $\begin{array}{c}0.0061 \\
(0.0166)\end{array}$ & $\begin{array}{c}0.79 \\
(0.534)\end{array}$ \\
\hline Trade openness & $\begin{array}{r}0.0252 * \\
(0.0138)\end{array}$ & $\begin{array}{r}-0.0130 \\
(0.0255)\end{array}$ & $\begin{array}{c}0.0224 \\
(0.0243)\end{array}$ & $\begin{array}{c}0.0188 \\
(0.0162)\end{array}$ & $\begin{array}{c}0.0254 \\
(0.0207)\end{array}$ & $\begin{array}{c}0.0082 \\
(0.0174)\end{array}$ & $\begin{array}{l}1.75 \\
(0.141)\end{array}$ \\
\hline Government spending & $\begin{array}{l}0.2125 * \star * \\
(0.0691)\end{array}$ & $\begin{array}{l}0.2408 * \star \\
(0.1097)\end{array}$ & $\begin{array}{l}0.2383^{* * *} \\
(0.0167)\end{array}$ & $\begin{array}{l}0.1733 * * * \\
(0.0619)\end{array}$ & $\begin{array}{l}0.1831^{* * *} \\
(0.0849)\end{array}$ & $\begin{array}{l}0.2235^{\star \star} \\
(0.0946)\end{array}$ & $\begin{array}{l}1.62 \\
(0.172)\end{array}$ \\
\hline $\begin{array}{l}\text { Representative } \\
\text { government }\end{array}$ & $\begin{array}{c}0.0158 \\
(0.0111)\end{array}$ & $\begin{array}{c}0.0022 \\
(0.0204)\end{array}$ & $\begin{array}{c}0.0174 \\
(0.0167)\end{array}$ & $\begin{array}{l}0.0164 \\
(0.0112)\end{array}$ & $\begin{array}{c}0.0157 \\
(0.0138)\end{array}$ & $\begin{array}{c}0.0259 \\
(0.0182)\end{array}$ & $\begin{array}{l}1.11 \\
(0.355)\end{array}$ \\
\hline Crisis & $\begin{array}{l}0.3908 * * * \\
(0.1948)\end{array}$ & $\begin{array}{l}1.4646^{\star} \\
(0.7716)\end{array}$ & $\begin{array}{l}0.6123 * \star \\
(0.2628)\end{array}$ & $\begin{array}{l}0.3439 * \star \\
(0.1731)\end{array}$ & $\begin{array}{c}0.3815 \\
(0.5765)\end{array}$ & $\begin{array}{c}0.1043 \\
(0.5244)\end{array}$ & $\begin{array}{c}0.12 \\
(0.974)\end{array}$ \\
\hline $\begin{array}{l}\text { Observations } \\
\mathrm{R}^{2}\end{array}$ & $\begin{array}{l}219 \\
0.099\end{array}$ & $\begin{array}{l}219 \\
0.120\end{array}$ & $\begin{array}{l}219 \\
0.072\end{array}$ & $\begin{array}{l}219 \\
0.059\end{array}$ & $\begin{array}{l}219 \\
0.081\end{array}$ & $\begin{array}{l}219 \\
0.026\end{array}$ & \\
\hline \multicolumn{8}{|c|}{ C. Lower- and lower-middle-income countries } \\
\hline $\begin{array}{l}\text { Financial } \\
\text { institutions } \\
\text { development }\end{array}$ & $\begin{array}{l}0.0709 * \star \\
(0.0331)\end{array}$ & $\begin{array}{l}0.0866 \\
(0.0802)\end{array}$ & $\begin{array}{c}0.0692 \\
(0.0569)\end{array}$ & $\begin{array}{c}0.0435 \\
(0.0324)\end{array}$ & $\begin{array}{c}0.0242 \\
(0.0349)\end{array}$ & $\begin{array}{c}0.0650 \\
(0.0847)\end{array}$ & $\begin{array}{l}5.78 \\
(0.001)\end{array}$ \\
\hline $\begin{array}{l}\text { Growth of GDP per } \\
\text { capita }\end{array}$ & $\begin{array}{l}-0.0395 \\
(0.0482)\end{array}$ & $\begin{array}{l}-0.0986 \\
(0.1471)\end{array}$ & $\begin{array}{l}-0.0716 \\
(0.1045)\end{array}$ & $\begin{array}{l}-0.0317 \\
(0.0594)\end{array}$ & $\begin{array}{l}-0.0114 \\
(0.0639)\end{array}$ & $\begin{array}{l}-0.0035 \\
(0.1500)\end{array}$ & $\begin{array}{l}0.15 \\
(0.962)\end{array}$ \\
\hline Inflation & $\begin{array}{l}0.0029 \\
(0.0127)\end{array}$ & $\begin{array}{l}0.0344 \\
(0.0371)\end{array}$ & $\begin{array}{l}0.0240 \\
(0.0263)\end{array}$ & $\begin{array}{l}0.0086 \\
(0.0151)\end{array}$ & $\begin{array}{l}-0.0053 \\
(0.0159)\end{array}$ & $\begin{array}{l}-0.0043 \\
(0.0321)\end{array}$ & $\begin{array}{l}2.06 \\
(0.009)\end{array}$ \\
\hline Trade openness & $\begin{array}{c}0.0001 \\
(0.0113)\end{array}$ & $\begin{array}{c}0.0020 \\
(0.0226)\end{array}$ & $\begin{array}{c}0.0037 \\
(0.0160)\end{array}$ & $\begin{array}{c}0.0063 \\
(0.0091)\end{array}$ & $\begin{array}{l}0.0044 \\
(0.0098)\end{array}$ & $\begin{array}{c}0.0017 \\
(0.0228)\end{array}$ & $\begin{array}{l}1.28 \\
(0.279)\end{array}$ \\
\hline Government spending & $\begin{array}{c}0.0143 \\
(0.0410)\end{array}$ & $\begin{array}{l}-0.0187 \\
(0.1028)\end{array}$ & $\begin{array}{l}-0.0043 \\
(0.0730)\end{array}$ & $\begin{array}{c}0.0171 \\
(0.0415)\end{array}$ & $\begin{array}{c}0.0339 \\
(0.0437)\end{array}$ & $\begin{array}{c}0.0311 \\
(0.1010)\end{array}$ & $\begin{array}{c}1.38 \\
(0.241)\end{array}$ \\
\hline $\begin{array}{l}\text { Representative } \\
\text { government }\end{array}$ & $\begin{array}{l}-0.0227 * \star \\
(0.0090)\end{array}$ & $\begin{array}{l}-0.0424^{*} \\
(0.0253)\end{array}$ & $\begin{array}{l}-0.0382 * \star \\
(0.0180)\end{array}$ & $\begin{array}{l}- \\
0.0320 * \star \star \\
(0.0102)\end{array}$ & $\begin{array}{l}-0.0264 * \star \\
(0.0110)\end{array}$ & $\begin{array}{l}-0.0080 \\
(0.0239)\end{array}$ & $\begin{array}{l}2.10 \\
(0.081)\end{array}$ \\
\hline Crisis & $\begin{array}{c}0.1951 \\
(0.2034)\end{array}$ & $\begin{array}{c}0.2248 \\
(0.4270)\end{array}$ & $\begin{array}{c}0.2221 \\
(0.3031)\end{array}$ & $\begin{array}{c}0.2181 \\
(0.1718)\end{array}$ & $\begin{array}{c}0.1848 \\
(0.1828)\end{array}$ & $\begin{array}{c}0.1126 \\
(0.4427)\end{array}$ & $\begin{array}{l}1.62 \\
(0.172)\end{array}$ \\
\hline Observations & 308 & 308 & 308 & 308 & 308 & 308 & \\
\hline $\mathrm{R}^{2}$ & 0.070 & 0.046 & 0.031 & 0.038 & 0.021 & 0.014 & \\
\hline $\begin{array}{l}\text { Countries are class } \\
\text { (1) reports panel } \\
\text { contain the coeffic } \\
\text { quantiles of the gi } \\
\text { during the period } 1\end{array}$ & ed accor & $\begin{array}{l}\text { to the } \\
\text { lts witl }\end{array}$ & Id Bar & $\begin{array}{l}2015 \text { inc } \\
y \text { and } t\end{array}$ & $\begin{array}{l}\text { group } \\
\text { effect }\end{array}$ & $\begin{array}{l}\text { Ssificat } \\
\text { Columns } \\
, 75 \text { and }\end{array}$ & $\begin{array}{l}\text { h. Column } \\
\text { ) to (6) } \\
\text { o percent } \\
\text { hual data }\end{array}$ \\
\hline
\end{tabular}


purports that the estimated slope coefficients for each variable are not statistically different across all the quantile estimates. The p-value for this test is given below the equality test value. $* * *, * *$ and $*$ indicate statistical significance at the $1 \%, 5 \%$ and $10 \%$ levels, respectively. 
Table 4

Financial markets development and income inequality by country income classification: dependent variable Gini coefficient

\begin{tabular}{|c|c|c|c|c|c|c|c|}
\hline & $(1)$ & $(2)$ & (3) & $(4)$ & (5) & $(6)$ & $(7)$ \\
\hline & $\begin{array}{c}\text { Fixed } \\
\text { effects }\end{array}$ & Q10 & Q25 & 250 & 275 & 290 & $\begin{array}{l}\text { Equality } \\
\text { test }\end{array}$ \\
\hline \multicolumn{8}{|c|}{ A. High-income countries } \\
\hline Financial markets & & & & & & & 1.02 \\
\hline development & $\begin{array}{l}0.0662 * * * \\
(0.0096)\end{array}$ & $\begin{array}{l}0.0777 * * * \\
(0.0139)\end{array}$ & $\begin{array}{l}0.0743 * \star \star \\
(0.0099)\end{array}$ & $\begin{array}{l}0.0695 * * * \\
(0.0085)\end{array}$ & $\begin{array}{l}0.0644 * * * \\
(0.0133)\end{array}$ & $\begin{array}{l}0.0605^{* * *} \\
(0.0140)\end{array}$ & $(0.396)$ \\
\hline $\begin{array}{l}\text { Growth of GDP per } \\
\text { capita }\end{array}$ & $\begin{array}{l}-0.0426 \\
(0.0795)\end{array}$ & $\begin{array}{l}-0.2479 * \star \\
(0.1173)\end{array}$ & $\begin{array}{l}-0.2018 * \star \\
(0.0842)\end{array}$ & $\begin{array}{l}-0.1382^{\star} \\
(0.0724)\end{array}$ & $\begin{array}{l}-0.0695 \\
(0.1127)\end{array}$ & $\begin{array}{l}-0.0357 \\
(0.1174)\end{array}$ & $\begin{array}{l}3.38 \\
(0.010)\end{array}$ \\
\hline Inflation & $\begin{array}{l}- \\
0.0411 * * * \\
(0.0134)\end{array}$ & $\begin{array}{l}-0.0245 \\
(0.0181)\end{array}$ & $\begin{array}{l}-0.0265 * \star \\
(0.0129)\end{array}$ & $\begin{array}{l}- \\
0.0293 * * * \\
(0.0111)\end{array}$ & $\begin{array}{l}-0.0324 * \\
(0.0174)\end{array}$ & $\begin{array}{l}-0.0331 * \\
(0.0172)\end{array}$ & $\begin{array}{l}1.83 \\
(0.124)\end{array}$ \\
\hline Trade openness & $\begin{array}{l}0.0382 * * * \\
(0.0094)\end{array}$ & $\begin{array}{l}0.0363 * * * \\
(0.0137)\end{array}$ & $\begin{array}{l}0.0357 * * * \\
(0.0098)\end{array}$ & $\begin{array}{l}0.0349 * * * \\
(0.0084)\end{array}$ & $\begin{array}{l}0.0340 * * * \\
(0.0132)\end{array}$ & $\begin{array}{l}0.0369 * \\
(0.0217)\end{array}$ & $\begin{array}{l}0.75 \\
(0.561)\end{array}$ \\
\hline Government spending & $\begin{array}{l}0.1636^{\star} \\
(0.0898)\end{array}$ & $\begin{array}{c}0.0066 \\
(0.1454)\end{array}$ & $\begin{array}{c}0.0208 \\
(0.1041)\end{array}$ & $\begin{array}{l}0.0404 \\
(0.0891)\end{array}$ & $\begin{array}{c}0.0616 \\
(0.1388)\end{array}$ & $\begin{array}{c}0.0332 \\
(0.1419)\end{array}$ & $\begin{array}{l}3.01 \\
(0.119)\end{array}$ \\
\hline $\begin{array}{l}\text { Representative } \\
\text { government }\end{array}$ & $\begin{array}{l}-0.0134 \\
(0.0159)\end{array}$ & $\begin{array}{l}-0.0305 \\
(0.02229)\end{array}$ & $\begin{array}{l}-0.0249 \\
(0.0164)\end{array}$ & $\begin{array}{l}-0.0171 \\
(0.0141)\end{array}$ & $\begin{array}{l}-0.0088 \\
(0.0220)\end{array}$ & $\begin{array}{l}-0.0076 \\
(0.0224)\end{array}$ & $\begin{array}{l}1.32 \\
(0.263)\end{array}$ \\
\hline Crisis & $\begin{array}{l}1.0564 * * * \\
(0.4573)\end{array}$ & $\begin{array}{l}-0.1022 \\
(0.4648)\end{array}$ & $\begin{array}{l}-0.0269 \\
(0.3329)\end{array}$ & $\begin{array}{c}0.0768 \\
(0.2853)\end{array}$ & $\begin{array}{l}1.1428 * \star \\
(0.4528)\end{array}$ & $\begin{array}{c}0.2276 \\
(0.4352)\end{array}$ & $\begin{array}{l}2.23 \\
(0.063)\end{array}$ \\
\hline Observations & 261 & 261 & 261 & 261 & 261 & 261 & \\
\hline $\mathrm{R}^{2}$ & 0.101 & 0.033 & 0.096 & 0.0085 & 0.027 & 0.018 & \\
\hline \multicolumn{8}{|c|}{ B. Upper middle-income countries } \\
\hline $\begin{array}{l}\text { Financial markets } \\
\text { development }\end{array}$ & $\begin{array}{l}-0.0462 * \star \\
(0.0277)\end{array}$ & $\begin{array}{l}-0.0898 * \star \\
(0.0441)\end{array}$ & $\begin{array}{l}- \\
0.0773 * * * \\
(0.0256)\end{array}$ & $\begin{array}{l}- \\
0.0568 * * * \\
(0.0208)\end{array}$ & $\begin{array}{l}-0.0555 * \star \\
(0.0271)\end{array}$ & $\begin{array}{l}-0.0314 \\
(0.0318)\end{array}$ & $\begin{array}{c}6.22 \\
(0.000)\end{array}$ \\
\hline $\begin{array}{l}\text { Growth of GDP per } \\
\text { capita }\end{array}$ & $\begin{array}{r}0.0744^{*} \\
(0.0452)\end{array}$ & $\begin{array}{r}0.0428 \\
(0.0967)\end{array}$ & $\begin{array}{c}0.0483 \\
(0.0582)\end{array}$ & $\begin{array}{c}0.0425 \\
(0.0482)\end{array}$ & $\begin{array}{c}0.0072 \\
(0.0571)\end{array}$ & $\begin{array}{l}-0.0584 \\
(0.0531)\end{array}$ & $\begin{array}{c}0.71 \\
(0.587)\end{array}$ \\
\hline Inflation & $\begin{array}{c}0.0125 \\
(0.0097)\end{array}$ & $\begin{array}{c}0.0278 \\
(0.0216)\end{array}$ & $\begin{array}{c}0.0193 \\
(0.0126)\end{array}$ & $\begin{array}{c}0.0151 \\
(0.0101)\end{array}$ & $\begin{array}{c}0.0098 \\
(0.0144)\end{array}$ & $\begin{array}{l}-0.0021 \\
(0.0166)\end{array}$ & $\begin{array}{l}1.08 \\
(0.370)\end{array}$ \\
\hline Trade openness & $\begin{array}{l}0.0199 \\
(0.0137)\end{array}$ & $\begin{array}{l}-0.0019 \\
(0.0247)\end{array}$ & $\begin{array}{l}-0.0015 \\
(0.0148)\end{array}$ & $\begin{array}{l}-0.0003 \\
(0.0120)\end{array}$ & $\begin{array}{l}0.0043 \\
(0.0149)\end{array}$ & $\begin{array}{c}0.0001 \\
(0.0181)\end{array}$ & $\begin{array}{l}1.43 \\
(0.225)\end{array}$ \\
\hline Government spending & $\begin{array}{l}0.2167 * * * \\
(0.0679)\end{array}$ & $\begin{array}{l}0.1724 \\
(0.1357)\end{array}$ & $\begin{array}{l}0.1610 * \star \\
(0.0804)\end{array}$ & $\begin{array}{l}0.1603 * * \\
(0.0659)\end{array}$ & $\begin{array}{l}0.1658 * \star \\
(0.0843)\end{array}$ & $\begin{array}{l}0.3115 * * * \\
(0.0930)\end{array}$ & $\begin{array}{l}4.67 \\
(0.001)\end{array}$ \\
\hline $\begin{array}{l}\text { Representative } \\
\text { government }\end{array}$ & $\begin{array}{l}0.0103 \\
(0.0112)\end{array}$ & $\begin{array}{c}0.0454 * \\
(0.0254)\end{array}$ & $\begin{array}{l}0.0333 * \star \\
(0.0150)\end{array}$ & $\begin{array}{l}0.0259 * \star \\
(0.0119)\end{array}$ & $\begin{array}{c}0.0199 \\
(0.0155)\end{array}$ & $\begin{array}{r}0.0345^{*} \\
(0.0201)\end{array}$ & $\begin{array}{l}1.57 \\
(0.184)\end{array}$ \\
\hline Crisis & $\begin{array}{l}0.9484 * * \\
(0.4491)\end{array}$ & $\begin{array}{l}0.3402 \\
(0.3558)\end{array}$ & $\begin{array}{r}0.7709 * \\
(0.4653)\end{array}$ & $\begin{array}{c}0.1529 \\
(0.1735)\end{array}$ & $\begin{array}{l}-0.1378 \\
(0.2312)\end{array}$ & $\begin{array}{c}0.3666 \\
(0.5799)\end{array}$ & $\begin{array}{l}2.02 \\
(0.091)\end{array}$ \\
\hline Observations & 221 & 221 & 221 & 221 & 221 & 221 & \\
\hline & 0.102 & 0.018 & 0.105 & 0.043 & 0.015 & 0.025 & \\
\hline \multicolumn{8}{|c|}{ C. Lower- and lower-middle-income countries } \\
\hline $\begin{array}{l}\text { Financial markets } \\
\text { development }\end{array}$ & $\begin{array}{l}0.0747 \star \star \star \\
(0.0258)\end{array}$ & $\begin{array}{l}0.0848 * \star \\
(0.0374)\end{array}$ & $\begin{array}{l}0.0782 * * \\
(0.0261)\end{array}$ & $\begin{array}{l}0.0697 * \star \star \\
(0.0177)\end{array}$ & $\begin{array}{l}0.0534 * \star \\
(0.0218)\end{array}$ & $\begin{array}{l}0.0777 * \star \\
(0.0336)\end{array}$ & $\begin{array}{l}1.39 \\
(0.239)\end{array}$ \\
\hline $\begin{array}{l}\text { Growth of GDP per } \\
\text { capita }\end{array}$ & $\begin{array}{l}-0.0162 \\
(0.0499)\end{array}$ & $\begin{array}{l}-0.0793 \\
(0.1182)\end{array}$ & $\begin{array}{l}-0.0543 \\
(0.0826)\end{array}$ & $\begin{array}{l}-0.0220 \\
(0.0560)\end{array}$ & $\begin{array}{c}0.0227 \\
(0.6598)\end{array}$ & $\begin{array}{c}0.0105 \\
(0.0905)\end{array}$ & $\begin{array}{l}1.52 \\
(0.195)\end{array}$ \\
\hline Inflation & $\begin{array}{c}0.0044 \\
(0.0126)\end{array}$ & $\begin{array}{l}0.0280 \\
(0.0308)\end{array}$ & $\begin{array}{c}0.0198 \\
(0.0215)\end{array}$ & $\begin{array}{c}0.0091 \\
(0.0146)\end{array}$ & $\begin{array}{l}-0.0009 \\
(0.0181)\end{array}$ & $\begin{array}{l}-0.0123 \\
(0.0309)\end{array}$ & $\begin{array}{l}1.70 \\
(0.150)\end{array}$ \\
\hline Trade openness & $\begin{array}{c}-0.0037 \\
(0.0119)\end{array}$ & $\begin{array}{c}0.0046 \\
(0.0194)\end{array}$ & $\begin{array}{c}0.0049 \\
(0.0136)\end{array}$ & $\begin{array}{c}0.0052 \\
(0.0092)\end{array}$ & $\begin{array}{c}0.0040 \\
(0.0115)\end{array}$ & $\begin{array}{l}-0.0094 \\
(0.0173)\end{array}$ & $\begin{array}{l}1.63 \\
(0.166)\end{array}$ \\
\hline Government spending & $\begin{array}{c}0.0419 \\
(0.0445)\end{array}$ & $\begin{array}{l}0.0387 \\
(0.0846)\end{array}$ & $\begin{array}{l}0.0041 \\
(0.0637)\end{array}$ & $\begin{array}{c}0.0442 \\
(0.0400)\end{array}$ & $\begin{array}{l}0.0476 \\
(0.0499)\end{array}$ & $\begin{array}{l}0.1170 * \\
(0.0668)\end{array}$ & $\begin{array}{l}2.75 \\
(0.028)\end{array}$ \\
\hline $\begin{array}{l}\text { Representative } \\
\text { government }\end{array}$ & $\begin{array}{l}-0.0281 * \star \\
(0.0092)\end{array}$ & $\begin{array}{l}0.0598 * \star \star \\
(0.0215)\end{array}$ & $\begin{array}{l}- \\
0.0517 \star \star \star \\
(0.0150)\end{array}$ & $\begin{array}{l}-0.0411^{\star \star} \\
(0.0102)\end{array}$ & $\begin{array}{l}-0.0316 * \star \\
(0.0127)\end{array}$ & $\begin{array}{l}-0.0099 \\
(0.1170)\end{array}$ & $\begin{array}{l}2.28 \\
(0.061)\end{array}$ \\
\hline Crisis & $\begin{array}{c}0.5744 \\
(0.4264)\end{array}$ & $\begin{array}{l}0.2549 \\
(0.3663)\end{array}$ & $\begin{array}{c}0.2509 \\
(0.2561)\end{array}$ & $\begin{array}{c}0.2547 \\
(0.1731)\end{array}$ & $\begin{array}{c}0.2254 \\
(0.2149)\end{array}$ & $\begin{array}{c}0.0960 \\
(0.2970)\end{array}$ & $\begin{array}{l}1.08 \\
(0.367)\end{array}$ \\
\hline Observations & 310 & 310 & 310 & 310 & 310 & 310 & \\
\hline $\mathrm{R}^{2}$ & 0.080 & 0.064 & 0.040 & 0.040 & 0.033 & 0.026 & \\
\hline \multicolumn{8}{|c|}{$\begin{array}{l}\text { Countries are classified according to the World Bank's } 2015 \text { income group classification. Column } \\
\text { (1) reports panel regression results with fixed country and time effects. Columns (2) to (6) } \\
\text { contain the coefficients of quantile estimates regressions for the 10, 25, 50, 75 and 90 percent } \\
\text { quantiles of the gini coefficient calculated as 5-year non-overlapping averages of annual data } \\
\text { during the period } 1971 \text { to 2015The equality test applied is the F-test where the null hypothesis } \\
\text { purports that the estimated slope coefficients for each variable are not statistically different }\end{array}$} \\
\hline
\end{tabular}


across all the quantile estimates. The p-value for this test is given below the equality test value. $* * *, * *$ and $*$ indicate statistical significance at the $1 \%, 5 \%$ and $10 \%$ levels, respectively. 